\title{
What's in a name and does CUI matter?
}

\author{
Martin Porcheron \\ Swansea University \\ Swansea, UK \\ m.a.w.porcheron@swansea.ac.uk
}

\begin{abstract}
In this paper, I provide some thoughts on the moniker that adorns our conference and technology: CUI. The name has proven to be problematic, as evidenced through many provocation papers in prior CUI conferences. The name presents a mismatch or a promise that is not necessarily fulfilled by the technology. But what of this? The name serves us well as a provocation itself, to think and reflect. However, we must still be cautious: to learn from the past, preoccupying ourselves with metaphor risks a poor user experience.
\end{abstract}

\section{CCS CONCEPTS}

- Human-centered computing $\rightarrow$ Natural language interfaces.

\section{KEYWORDS}

conversational user interfaces, voice interfaces, speech interfaces, chatbots

\section{ACM Reference Format:}

Martin Porcheron. 2021. What's in a name and does CUI matter?. In 3rd Conference on Conversational User Interfaces (CUI '21), July 27-29, 2021, Bilbao (online), Spain. ACM, New York, NY, USA, 3 pages. https://doi.org/10. $1145 / 3469595.3469619$

\section{INTRODUCTION}

Conversational User Interfaces (CUIs) have arguably been never better (in terms of efficacy, efficiency, aesthetics, availability, affordability, and so on) and the pace of innovation is rapid with industry and academics working around the world to understand their use and to improve the experience for everyone. Accompanying this, though, is bountiful critique-both in and outside of academia-for the technology's various embodiments. Some of this critique even focuses on what we call these sorts of technologies. I want to stress that, holistically taken, I believe this should all be regarded in a positive light. It is these critiques that can provoke introspection, revisionism, and innovation. But I want to take a moment to reflect on just a modicum of this debate and to add what represents my current state of mind ${ }^{1}$ about the CUI community, and its lust for provocation, especially about the name CUI.

As such, this provocation paper seeks not to mimic existing stipulations and perspectives of current technological limitations, to highlight new problems per sé, or to even counter any existing

\footnotetext{
${ }^{1}$ At the time of writing, April 2021. My thoughts are fluid and open to change. I eagerly look forward to discussing this further at CUI.

CUI '21, fuly 27-29, 2021, Bilbao (online), Spain

(c) 2021 Copyright held by the owner/author(s). Publication rights licensed to ACM.

This is the author's version of the work. It is posted here for your personal use. Not for redistribution. The definitive Version of Record was published in $3 \mathrm{rd}$ Conference on Conversational User Interfaces (CUI '21), July 27-29, 2021, Bilbao (online), Spain, https://doi.org/10.1145/3469595.3469619.
}

critique. Instead, this paper embraces critique within the CUI community of our name. Before any reader-instigated excitement that this paper offers a literature review to feast upon: it does not. This year represents the third annual CUI conference, and thus it is far too premature for a literature view of any substantive rigour ${ }^{2}$. In this paper, I turn to reflect on only a subset of provocation papers published in the proceedings of CUI '19 and CUI '20-which certainly only represents a fraction of the debate related issues with AI-based technologies, much of which can be found elsewhere (e.g., [19]). I want to turn to the critique of the name of our community, and the categorical moniker: the Conversational User Interface.

\section{THE PROBLEM WITH CUI}

As per The Pointy-Haired Boss in the first episode of the television series Dilbert, the product name is the most important thing ${ }^{3}$. As such, the natural commencement for many CUI provocation papers is to take aim at the name of the technology and community. The $C U I$ name exists to represent the union of both textual and auditory-driven interfaces, both forms of which have themselves a multitude of names-e.g., chatbots, speech interfaces, voice user interfaces, voice-activated personal assistants, and so on. This leads to a keyword soup in every CUI paper though. But what's wrong with calling this amalgam of technology a CUI? Well, for the first two years of CUI, critiques have challenged the use of the term 'conversational' [12], that wake words are unnatural [1], that the multimodality of interhuman conversation implies technology should attend to this multimodality [15], or that such interfaces should be embodied themselves [7]. Chavez-Sanchez et al. [2] also ask "How do we understand conversations?", arguing we need to consider the full gamut of vocal interaction when designing technologies for auditory interaction. This is perhaps not too surprising: conversation is a term most of us will understand, in part because we are all mostly competent conversationalists. Even the most rudimentary examination of any CUI technology will elucidate that this technology does not exhibit the full catalogue of features a typical adult human can inject into their talk. In fact, you could argue the technology often relies upon a crude approximation of conversation, and perhaps the denomination of 'request-response' is more realistic [11].

However, conversational is not the only word to have provoked the CUI community; Edlund [5] masterly route through their provocation paper to an issue of HCI's treatment of the word interface, and crucially the idea of equating of "interaction with manipulation of an interface", suggesting a move beyond the regimented framing of interfaces, linking up with critiques of limiting ourselves to

\footnotetext{
${ }^{2}$ If you are looking for a literature review of speech-related technologies in HCI, I recommend the comprehensive review by Clark et al. [4].

${ }^{3}$ The joke being, Dilbert thinks that deciding what the product will be is more important.
} 
'conversation'. Indeed, rarely is a CUI just an interface, but a more complete system combining Internet of Things technologies, Machine Learning-driven decision making systems, and often remote information retrieval systems. Simpson [17] seemingly concurs with this, calling for researchers to move beyond GUI conceptualisations of CUIs. It seems that an interface downplays the needs for researchers and engineers to focus on the bigger picture with these technologies. CSI: NY's use of the term 'GUI interface' to 'track an IP' garnered mockery in the 2000s and, indeed, it is easy to fall into this trap ourselves when describing a more involved system as merely an interface.

Right now, the elephant in the room is the word user, perhaps the least critiqued so far within CUI itself. In CUI, Sankaran et al. [14] respond to notions of human autonomy being lost to machines, raising questions of who is the user, and what it is they do? Much of the debate about user comes from outside of CUI though. Seberger [16], with regards to the Internet of Things, asks if the technology is ubiquitous, aren't we all users? Or staying within the realm of CUIs (but admittedly outside of the CUI conference), in reviewing the underlying core principles of human-centred design, Giaccardi and Redström [8] locate a need to consider more-than-human design, arguing that "we still tend to place the one-to-one interaction, the sequence of touchpoints a user will encounter and interact with, at the center of how we give form and expression to the design" [8]. Yet, increasingly algorithmic influences challenge notions of agency, the modus operandi for design thinking needs to evolve. CUIs are increasingly used as control points for Machine Learning enhanced Internet of Things technologies, and act as a gateway to algorithmically-influenced interactions. As such, we must also consider our understanding of design, and our relationship with users, moving beyond existing at-the-coalface idealisations of technology to embrace the reality of systems ostensibly possessing agency.

The problem, as Reeves put it, is that "CUIs trade on various category mistakes" [12]. Each word in CUI carries with it sociotechnical expectations, and in turn each engenders provocation.

\section{THE PROBLEM WITH CUIS}

Thus, perhaps I could have concluded with that summary but I want to go further than simply treat the problem with the CUI moniker as perhaps its mismatch between the reality, the imagined technologies evoked by the name, the limits that some see that name placing on designers' thoughts about the capabilities of the technology, and the disparity between the terms used by designers and the commonly and academically understood meanings of those words. Firstly, I think we must be cautious in avoiding the previous mistakes of the AI community for we risk another AI winter [6] beset with over-promises and under-deliverance. The 'space race' of building ever-more realistic voices and devices capable of conversing as much as possible [9], risks sidelining efforts to understand the needs and benefits of CUIs-we must not eschew functional improvements in favour of glitz. The promises of a fully-conversant AI around the corner are only palatable for so long and investors will tire, and academics will move on (as they have done before!).

I also turn to the wisdom of Suchman's Plans and Situated Actions to more eloquently demonstrate this problem space and how we can (should?) reorient our provocations. In the book, Suchman focuses on the challenges users encounter with an intelligent photocopier assistant, and concludes that people are "... left to contend with the disparity between the hypothetical sequence of events on which the design is based, and the action's actual course". Bringing this to CUIs, the name, the promises, the dreams of what a CUI should be, what we (attempt to) imbue in their design to make them 'better' becomes the metaphors and the things people need to navigate to use the device. We must not ignore the fundamentals of what we know about user experience, that " $[t]$ he first requirement for an exemplary user experience is to meet the exact needs of the customer, without fuss or bother" [10]. CUIs are a means to an end: they play our music, they manage alarms, they handle our todo lists. When we become preoccupied with what a CUI is, isn't, should be, shouldn't be, could be, and possibly couldn't be, it is the 'end users'-the family in their kitchen [11], the visually impaired person in their living room [13], the cyclist on their bike-who wants a specific task to be completed, but must contend with designerly concerns of adequate metaphors. A computer inserting a periodical um [3] or a humanistic voice responding with chirpy affirmations is amazing, but what of 'getting things done'? Design is much more than the aesthetic and yet, at least ostensibly, aesthetic is often treated as the primary marker of a CUI's capability.

\section{THE PROBLEM?}

Now, before anyone assumes this paper to be an anti-progress rant, the above is not to say we should not pursue better text-to-speech voices or better technologies for dealing with the disfluencies of talk. Each innovation opens the door to new possibilities, new opportunities, and excites people to join our community to make the world a better place. Better recognition means users can certainly complete their tasks more easily and CUIs that sounds more aesthetically pleasing may be more joyful to use (which is, of course, all part of the user experience). Improved technology can be a betterment for all of humanity.

This provocation is also not to say that we must not provoke further. We must, and we must go deeper. But I feel we must move beyond the naming of 'our' technology and community, and what we can or cannot achieve. The moniker of CUI is perhaps not the most descriptive or accurate of product names, but I ask, what is in a name anyway? The mainstay of intellectual debate about the name is valid and validated in my opinion. It also continues to provide momentum for researchers, designers, and engineers to critique. I wonder, what can we do if we see beyond the name. What if we move beyond the obvious self-provocation of CUI, and instead occupy ourselves with making the world better.

In concluding this abridged reflection, I celebrate the community of provocateurs. The CUI conference provocations track is about making sparks, inspiring thoughts, and building connections. To this aim, it succeeds, and I say that together we can move forward and ask not what CUI does for the technology or the community, but what we can do for the world? 


\section{REFERENCES}

[1] Shashank Ahire and Michael Rohs. 2020. Tired of Wake Words? Moving Towards Seamless Conversations with Intelligent Personal Assistants. In Proceedings of the 2nd Conference on Conversational User Interfaces (Bilbao, Spain) (CUI '20). ACM New York, NY, USA, Article 20, 3 pages. https://doi.org/10.1145/3405755.3406141

[2] Fermin Chavez-Sanchez, Gloria Adriana Mendoza Franco, Gloria Angelica Martínez de la Peña, and Erick Iroel Heredia Carrillo. 2020. Beyond What is Said: Looking for Foundational Principles in VUI Design. In Proceedings of the 2nd Conference on Conversational User Interfaces (Bilbao, Spain) (CUI '20). ACM, New York, NY, USA, Article 28, 3 pages. https://doi.org/10.1145/3405755.3406145

[3] Brian X. Chen and Cade Metz. 2019. Google's Duplex Uses A.I. to Mimic Humans (Sometimes). https://www.nytimes.com/2019/05/22/technology/personaltech/aigoogle-duplex.html

[4] Leigh Clark, Philip Doyle, Diego Garaialde, Emer Gilmartin, Stephan Schlögl, Jens Edlund, Matthew Aylett, João Cabral, Cosmin Munteanu, Justin Edwards, and Benjamin R Cowan. 2019. The State of Speech in HCI: Trends, Themes and Challenges. Interacting with Computers 31, 4 (09 2019), 349-371. https://doi.org/10.1093/iwc/iwz016 arXiv:https://academic.oup.com/iwc/articlepdf/31/4/349/33525046/iwz016.pdf

[5] Jens Edlund. 2019. Shoehorning in the Name of Science. In Proceedings of the 1st International Conference on Conversational User Interfaces (Dublin, Ireland) (CUI '19). ACM, New York, NY, USA, Article 8, 3 pages. https://doi.org/10.1145/ 3342775.3342794

[6] Luciano Floridi. 2020. AI and Its New Winter: from Myths to Realities. Philosophy \& Technology 33, 1 (2020), 1-3. https://doi.org/10.1007/s13347-020-00396-6

[7] Mary Ellen Foster. 2019. Face-to-Face Conversation: Why Embodiment Matters for Conversational User Interfaces. In Proceedings of the 1st International Conference on Conversational User Interfaces (Dublin, Ireland) (CUI '19). ACM, New York, NY, USA, Article 13, 3 pages. https://doi.org/10.1145/3342775.3342810

[8] Elisa Giaccardi and Johan Redström. 2020. Technology and More-Than-Human Design. Design Issues 36, 4 (09 2020), 33-44. https://doi.org/10.1162/desi a 00612

[9] Chandra Khatri, Anu Venkatesh, Behnam Hedayatnia, Raefer Gabriel, Ashwin Ram, and Rohit Prasad. 2018. Alexa Prize-State of the Art in Conversational AI. AI Magazine 39, 3 (2018), 40-55.
[10] Don Norman and Jakob Nielsen. [n.d.]. The Definition of User Experience (UX). https://www.nngroup.com/articles/definition-user-experience/

[11] Martin Porcheron, Joel E Fischer, Stuart Reeves, and Sarah Sharples. 2018. Voice Interfaces in Everyday Life. In Proceedings of the 2018 ACM Conference on Human Factors in Computing Systems (CHI '18). ACM, New York, NY, USA, Article 640, 12 pages. https://doi.org/10.1145/3173574.3174214

[12] Stuart Reeves. 2019. Conversation Considered Harmful?. In Proceedings of the 1st International Conference on Conversational User Interfaces (Dublin, Ireland) (CUI '19). ACM, New York, NY, USA, Article 10, 3 pages. https://doi.org/10.1145/ 3342775.3342796

[13] Gisela Reyes-Cruz, Joel E. Fischer, and Stuart Reeves. 2020. Reframing Disability as Competency: Unpacking Everyday Technology Practices of People with Visual Impairments. In Proceedings of the 2020 CHI Conference on Human Factors in Computing Systems (Honolulu, HI, USA) (CHI '20). ACM, New York, NY, USA, 1-13. https://doi.org/10.1145/3313831.3376767

[14] Supraja Sankaran, Chao Zhang, Mathias Funk, Henk Aarts, and Panos Markopoulos. 2020. Do I Have a Say? Using Conversational Agents to Re-Imagine HumanMachine Autonomy. In Proceedings of the 2nd Conference on Conversational User Interfaces (Bilbao, Spain) (CUI '20). ACM, New York, NY, USA, Article 18, 3 pages. https://doi.org/10.1145/3405755.3406135

[15] Stefan Schaffer and Norbert Reithinger. 2019. Conversation is Multimodal: Thus Conversational User Interfaces Should Be as Well. In Proceedings of the 1st International Conference on Conversational User Interfaces (Dublin, Ireland) (CUI '19). ACM, New York, NY, USA, Article 12, 3 pages. https://doi.org/10.1145/3342775. 3342801

[16] John S. Seberger. 2021. Reconsidering the user in IoT: the subjectivity of things. Personal and Ubiquitous Computing (2021), 9 pages. https://doi.org/10.1007/ s00779-020-01513-0

[17] James Simpson. 2020. Are CUIs Just GUIs with Speech Bubbles?. In Proceedings of the 2nd Conference on Conversational User Interfaces (Bilbao, Spain) (CUI '20). ACM, New York, NY, USA, Article 23, 3 pages. https://doi.org/10.1145/3405755.3406143

[18] Lucy A Suchman. 1985. Plans and Situated Actions: The Problem of Human Machine Communication (1 ed.). Cambridge University Press, Cambridge, UK. 220 pages.

[19] Jane Wakefield. 2021. AI: Ghost workers demand to be seen and heard. https: //www.bbc.co.uk/news/technology-56414491 